\title{
BMJ Open Potential economic consequences of a cardioprotective agent for patients with myocardial infarction: modelling study
}

\author{
Talitha I Verhoef, ${ }^{1}$ Stephen Morris, ${ }^{1}$ Anthony Mathur, ${ }^{2,3}$ Mervyn Singer ${ }^{4}$
}

To cite: Verhoef TI, Morris S, Mathur A, et al. Potential economic consequences of a cardioprotective agent for patients with myocardial infarction: modelling study. BMJ Open 2015;5:e008164. doi:10.1136/bmjopen-2015008164

\section{- Prepublication history} and additional material is available. To view please visit the journal (http://dx.doi.org/ 10.1136/bmjopen-2015008164).

Received 11 March 2015 Revised 20 October 2015 Accepted 26 October 2015

\section{(a) CrossMark}

${ }^{1}$ Department of Applied Health Research, University College London, London, UK ${ }^{2}$ Department of Cardiology, London Chest Hospital, Barts Health NHS Trust, London, UK

${ }^{3}$ Barts Health NIHR Cardiovascular Biomedical Research Unit, London Chest Hospital, Barts Health NHS Trust, London, UK ${ }^{4}$ Division of Medicine, Bloomsbury Institute of Intensive Care Medicine, University College London, London, UK

Correspondence to Dr Talitha I Verhoef; t.verhoef@ucl.ac.uk

\section{ABSTRACT}

Objective: To investigate the cost-effectiveness of a hypothetical cardioprotective agent used to reduce infarct size in patients undergoing percutaneous coronary intervention (PCI) after anterior ST-elevation myocardial infarction.

Methods: Design: A cost-utility analysis using a Markov model. Setting: The National Health Service in the UK. Patients: Patients undergoing PCI after anterior ST-elevation myocardial infarction. Interventions: A cardioprotective agent given at the time of reperfusion compared to no cardioprotection. We assumed the cardioprotective agent (given at the time of reperfusion) would reduce the risk and severity of heart failure (HF) after $\mathrm{PCl}$ and the risk of mortality after $\mathrm{PCl}$ (with a relative risk ranging from 0.6 to 1 ). The costs of the cardioprotective agent were assumed to be in the range $£ 1000-4000$. Main outcome measures: The incremental costs per quality-adjusted life-year (QALY) gained, using 95\% Cls from 1000 simulations.

Results: Incremental costs ranged from $£ 933$ to $£ 3820$ and incremental QALYs from 0.04 to 0.38 . The incremental cost-effectiveness ratio (ICER) ranged from $£ 3311$ to $£ 63480$ per QALY gained. The results were highly dependent on the costs of a cardioprotective agent, patient age, and the relative risk of $\mathrm{HF}$ after $\mathrm{PCI}$. The ICER was below the willingness-to-pay threshold of $£ 20000$ per QALY gained in $71 \%$ of the simulations.

Conclusions: A cardioprotective agent that can reduce the risk of $\mathrm{HF}$ and mortality after $\mathrm{PCI}$ has a high chance of being cost-effective. This chance depends on the price of the agent, the age of the patient and the relative risk of $\mathrm{HF}$ after $\mathrm{PCl}$.

\section{INTRODUCTION}

Acute myocardial infarction (AMI) is a major cause of mortality and morbidity, even with the use of early reperfusion strategies such as percutaneous coronary intervention (PCI). In case of AMI, a coronary artery becomes occluded, causing myocardial ischaemia, which in its turn causes myocardial necrosis. The extent of this necrosis, the infarct size, is

\section{Strengths and limitations of this study}

A strength of this study is that a model is presented that can be used for any potential cardioprotective agent to study the economic consequences of implementing this agent in clinical practice. If the effect of the new drug on the incidence of heart failure after $\mathrm{PCl}$ is known, this model can be updated to present a more precise estimate of the cost-effectiveness of this drug.

- The main limitation of this study is that it is based on a hypothetical drug. There are currently not enough data available on the long-term effectiveness of any of the potential cardioprotective agents.

- We also assumed that the cardioprotective agent would only reduce the risk and severity of heart failure and not the risk of other clinical major adverse cardiac events, such as recurrent infarction. In reality, a cardioprotective agent might have an effect on all such events. However, by only taking into account heart failure risk we present a conservative estimate of the costeffectiveness. A cardioprotective agent that will also decrease the risk of other major adverse cardiac events is expected to have a higher chance to be cost-effective.

a major determinant of mortality and morbidity after AMI. After AMI blood flow in the ischaemic myocardium should therefore be restored as soon as possible to minimise infarct size and associated complications. PCI is frequently used to accomplish this but, may itself cause injury to the myocardium as a consequence of the restoration in blood flow with generation of oxidative stress. The efficacy of reperfusion therapy is often assessed by measuring the infarct size using serum markers or MRI. Cardioprotection could be used at the time of reperfusion to reduce reperfusion injury and further decrease myocardial necrosis. Cardioprotection can be defined as any strategy to preserve the heart by reducing or even preventing myocardial damage. ${ }^{1}$ Many cardioprotective strategies 
have been developed and tested to reduce reperfusion injury and decrease infarct size..$^{2-4}$ These include postconditioning, remote ischaemic conditioning, intravenous cooling and various pharmacological agents. None of these strategies has yet translated into clinical practice; some did not have the desired effect in clinical trials while others are still in development. ${ }^{2-4}$

The longer term consequences of AMI are not only caused by the direct structural damage inflicted by the infarction, but also by secondary changes in the size and shape of the myocardium (ventricular remodelling). This can lead to ventricular dysfunction with subsequent heart failure (HF), long-term morbidity and a shortened lifespan. ${ }^{5}$ By reducing infarct size, cardioprotective agents potentially decrease the incidence and severity of HF after AMI, thereby reducing long-term morbidity which might carry significant economic consequences. Such consequences have not yet been studied. The aim of this study is to investigate the cost-effectiveness of a hypothetical cardioprotective agent used to reduce infarct size in patients undergoing PCI after anterior ST-elevation myocardial infarction (STEMI).

\section{METHODS}

\section{Model structure}

A Markov model was developed to assess the potential clinical effect and the economic consequences of a cardioprotective agent. It is expected that a reduced infarct size will affect the risk and severity of HF after STEMI. We conservatively assumed that the cardioprotective agent would only reduce the risk and severity of $\mathrm{HF}$ and not the risk of other clinical major adverse cardiac events, such as recurrent infarction. Figure 1 depicts the different health states in the Markov model. After PCI a patient could stay alive without HF, develop HF (in any of the four New York Heart Association (NYHA) classes) or die. Other than death, which is an absorbing state, patients either stay in their health state or move to another state

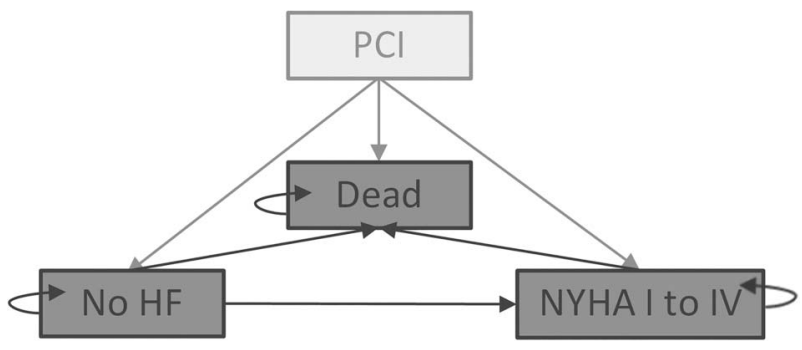

Figure 1 Health states in the Markov model. After PCI, patients could move to any of the health states (light grey arrows). In subsequent cycles, patients could stay in the health state or move to another health state (dark grey arrows). In the model, patients are unable to recover from having HF, but could move up and down between the different NYHA classes or stay without symptoms (NYHA class 1). Patients who did not have HF directly after $\mathrm{PCI}$ may develop HF later. HF, heart failure; NYHA, New York Heart Association; $\mathrm{PCl}$, percutaneous coronary intervention. in monthly cycles. In the model, patients are unable to recover from having HF, but could move up and down between the different NYHA classes or stay without symptoms (NYHA class 1). Patients who did not have HF directly after PCI may develop HF later. Transitions in any cycle were dependent on time since the start of the model, but not on what happened prior to that cycle ( past health states or how long they have been in a particular state).The model was run over a lifetime horizon.

\section{Clinical input \\ Natural disease history}

A literature search was performed to collect relevant data to populate the model. In our base case, patients were 65 years old when having PCI. Clinical outcome after PCI varies among different patient groups. Mortality after PCI has been reported between $3.4 \%^{7}$ to $7.8 \%,{ }^{8}$ and the incidence of $\mathrm{HF}$ from $5 \%{ }^{9}$ to almost $30 \% .{ }^{10}{ }^{11}$ We assumed that mortality after PCI would be $3.4 \%$ and the incidence of $\mathrm{HF} 10 \%$. Late onset $\mathrm{HF}$ would occur in $5 \%$ of patients in the first month after PCI, and a further $0.5 \%$ until 2 years after PCI. ${ }^{9} 11$ Clinical input parameters are shown in table 1 . Transition probabilities between the different NYHA classes were derived from a randomised trial ${ }^{12}$ as in previous cost-effectiveness studies of $\mathrm{HF}$ treatment ${ }^{13}{ }^{14}$ (table 2). Age-specific long-term mortality data of myocardial infarction patients were used to determine the mortality of patients in the 'No HF' or 'NYHA class I' state. ${ }^{15}$ This was done by applying monthly transition probabilities for mortality to all survivors of the previous month. An excess mortality risk was applied to patients in the NYHA class II to IV states $(0.26-0.72 \%$ if not hospitalised, $1.09-5.33 \%$ if hospitalised, see table 1). These data, as well as the probability of hospitalisation in the different NYHA classes, were derived from a costeffectiveness study on the treatment of $\mathrm{HF}^{13}$

\section{Efficacy of hypothetical cardioprotective agent}

As the analysis is based on a hypothetical cardioprotective agent, no data on effectiveness were currently available for use in this model. We assumed that the drug would affect the incidence of $\mathrm{HF}$ and mortality directly after PCI, as well as the incidence of new HF or worsening of existing HF in the 6 months following PCI. In previous studies on the effectiveness of cardioprotective strategies, varying results were found. In a study on the efficacy of adenosine in AMI it was found that 1 month mortality was reduced from $9.2 \%$ to $5.2 \%(\mathrm{p}=0.014)$ and 6-month mortality was reduced from $11.2 \%$ to $7.3 \%$ in patients receiving early reperfusion. ${ }^{21} \mathrm{~A}$ small reduction in in-hospital HF was seen, although this was not statistically significant $(3.2 \%$ vs $4.0 \%, \mathrm{p}=0.59)$. A larger reduction in $\mathrm{HF}$ was seen for ischaemic postconditioning in patients treated with PCI $(27 \%$ vs $46 \%, \mathrm{p}=0.048) .{ }^{22}$ In the current study we therefore assumed a relative risk of these events in the intervention group in the range of $60-100 \%$ (point estimate $80 \%$ ). 
Table 1 Input parameters

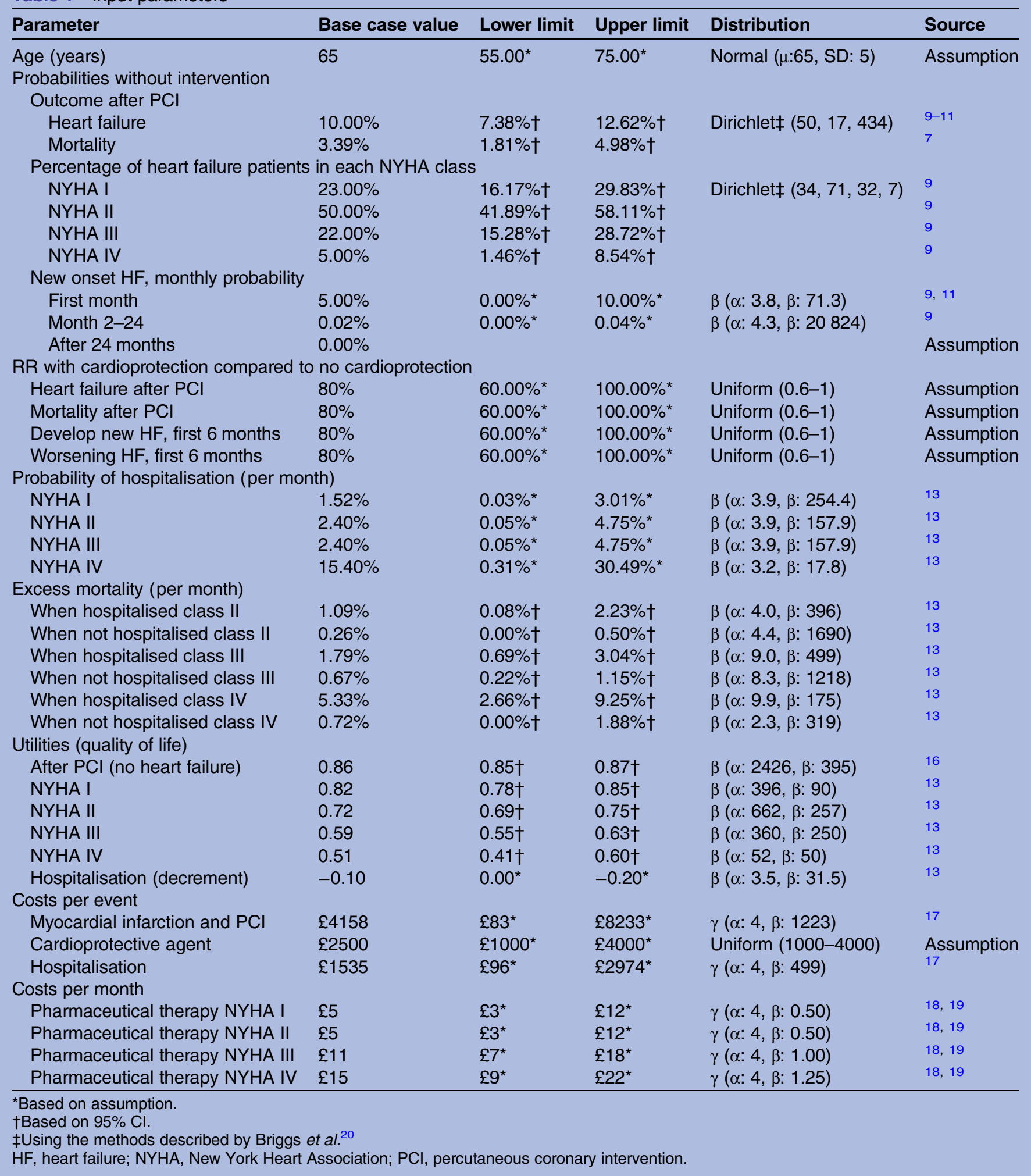

Health utility

The health utility after PCI, without HF, was 0.86 in our model based on results from the EuroQol Questionnaire as in previous studies. ${ }^{1623}$ Patients with HF had a lower quality of life, depending on the severity of the disease, varying from 0.82 in NYHA class I to 0.51 in NYHA class IV. ${ }^{13}$ When a patient was hospitalised, a temporary decrement of 0.10 was applied in the month following hospitalisation. $^{13} 14$

Costs

The perspective of this cost-effectiveness analysis was the cost to the National Health Service (NHS). Costs of AMI, PCI and HF events were derived from the NHS 
Table 2 Monthly transition probabilities among NYHA class $^{12-14}$

\begin{tabular}{lcccc}
\hline $\begin{array}{l}\text { From } \downarrow \\
\text { to } \rightarrow\end{array}$ & $\begin{array}{l}\text { NYHA I } \\
(\%)\end{array}$ & $\begin{array}{l}\text { NYHA II } \\
(\%)\end{array}$ & $\begin{array}{l}\text { NYHA III } \\
(\%)\end{array}$ & $\begin{array}{l}\text { NYHA IV } \\
(\%)\end{array}$ \\
\hline NYHA I & 97.70 & 1.90 & 0.40 & 0.00 \\
NYHA II & 0.80 & 98.10 & 1.00 & 0.10 \\
NYHA III & 0.00 & 3.40 & 96.00 & 0.60 \\
NYHA IV & 0.00 & 0.00 & 5.50 & 94.50 \\
\hline
\end{tabular}

Values were varied in the probabilistic sensitivity analysis using Dirichlet distributions. ${ }^{20}$

NYHA, New York Heart Association.

reference cost schedule 2012/2013. ${ }^{17}$ The monthly costs of the pharmaceutical treatment of HF were calculated using information on drug use in the different NYHA classes from a previous cost-effectiveness analysis ${ }^{19}$ and applying 2014 National formulary unit prices. ${ }^{18}$ We assumed that the drug would be administered at the time of reperfusion and would not affect the length of stay in the hospital or the need for diagnostic tests. For the cost of a new cardioprotective agent we assumed a one-off, one-time treatment of $£ 2500$ and because this is a hypothetical agent, we varied this over a wide range (£1000-£4000). Future costs and effects were discounted at an annual rate of $3.5 \%$.

\section{Analysis}

The main outcome of our analysis was the incremental costs per quality-adjusted life-year (QALY) gained which is the recommended cost-effectiveness measure for economic evaluations in England. ${ }^{24}$ Because there are currently no data available on the effect and costs of a cardioprotective agent, these parameters were varied over a wide range. To assess the uncertainty of the model we undertook several sensitivity analyses. First, all parameters, such as the cost of a cardioprotective agent, the cost of hospitalisation, the relative risk of HF with a cardioprotective agent, the relative risk of mortality after PCI with a cardioprotective agent, and the incidence of HF after PCI were varied in a one-way sensitivity analysis. Upper and lower limits were based on 95\% CIs if available, otherwise assumptions about the range were made. For some parameters, such as the '\% of patients with HF in each NYHA class', when one value was changed in the sensitivity analyses, the other values were changed simultaneously so that the sum would always be $100 \%$ (adding or subtracting the change in the parameter that was tested from the largest category). Second, the price and effect of a cardioprotective agent were varied simultaneously in a two-way sensitivity analysis to identify combinations of these values at which the ICER would be below $£ 20000$ per QALY gained. Lastly, a probabilistic sensitivity analysis was carried out, drawing random samples from the probability distributions of all parameters in 1000 simulations, using the distributions in table 1 . The proportion of times the ICER was less than the cost-effectiveness threshold was calculated for different values of the cost-effectiveness threshold, ranging from $£ 0$ to $£ 50000$, and presented graphically using cost-effectiveness acceptability curves.

\section{RESULTS}

\section{Base case}

As shown in figure 2, the percentage patients without HF decreased overtime, while the percentage of patients with HF initially increased, but decreased after the first year as more patients died. When a relative risk of $80 \%$ was used for events in users of a cardioprotective agent, the number of patients without HF was approximately $3 \%$ higher when a cardioprotective agent was used and the number of patients with $\mathrm{HF}$ approximately $2 \%$ lower. The percentage of patients who died was approximately $1 \%$ lower with a cardioprotective agent.

From the 1000 simulations, a 95\% confidence range could be calculated. The costs without use of a cardioprotective agent ranged from $£ 1793$ to $£ 10963$ (mean £5163) and QALYs ranged from 3.95 to 12.89 (mean 8.34). Patients lived on average 9.60 (SD: 2.39) years after STEMI. With the use of a cardioprotective agent, costs ranged from £3702 to £13 190 (mean £7498) and QALYs from 4.01 to 13.12 (mean 8.52). Patients were expected to live on average 9.77 (SD: 2.45) years after STEMI. Incremental costs were $£ 933$ to $£ 3820$ (mean $£ 2334$ ) and incremental QALYs 0.04 to 0.38 (mean 0.18). The ICER ranged from £3311 to 63480 per QALY gained (mean $£ 13014$ ). The results are summarised in table 3 .

\section{Sensitivity analysis}

As expected, the costs of a cardioprotective agent and the relative risk of $\mathrm{HF}$ after PCI with a cardioprotective agent had a large influence on the cost-effectiveness results in our one-way sensitivity analysis. If the costs of a cardioprotective agent would be $£ 1000$, the ICER would be $£ 4878$, while if the costs would be $£ 4000$ the ICER would be $£ 22004$ per QALY gained. Other factors that had a large influence on the ICER were age and relative

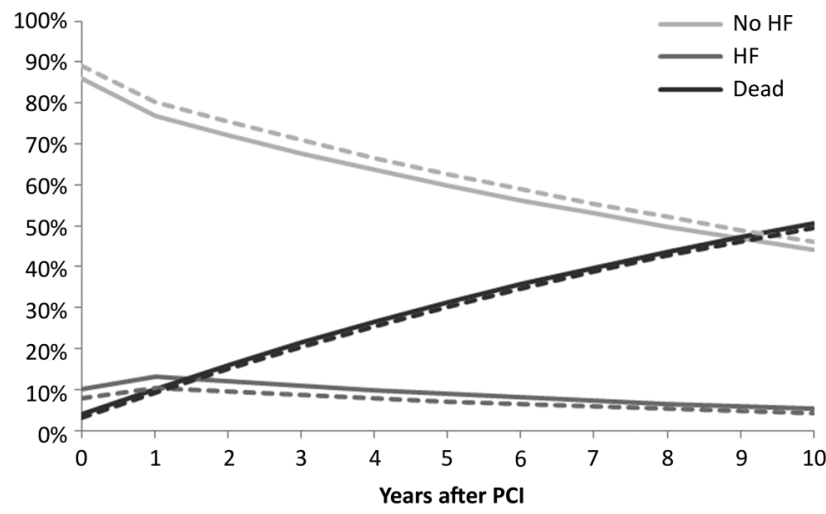

Figure 2 Percentage of patients with or without HF or who died. Solid line: no cardioprotection, dashed line: with cardioprotective agent. HF, heart failure; $\mathrm{PCl}$, percutaneous coronary intervention. 
Table 3 Results (95\% Cls) from the 1000 simulations

\begin{tabular}{llll}
\hline & Costs $(£)$ & QALYs & ICER (£/QALY) \\
\hline No cardioprotection & $£ 5163(1793$ to 10963$)$ & $8.34(3.95$ to 12.89$)$ & \\
Cardioprotective agent & $£ 7498(3702$ to 13190$)$ & $8.52(4.01$ to 13.12$)$ & £13 014 (3311 to 63 480) \\
Increment & $£ 2334(933$ to 3820) & $0.18(0.04$ to 0.38) &
\end{tabular}

risk of mortality after PCI with a cardioprotective agent. The ICER would exceed a threshold of $£ 20000$ in patients older than 70 and $£ 30000$ in patients older than 74 . The effect of uncertainty around several key parameters on the ICER is shown in the tornado diagram in figure 3.

When the price and effect of a cardioprotective agent were varied simultaneously we found that if the relative risk of the cardioprotective agent would be 0.6 (preventing $40 \%$ of $\mathrm{HF}$ cases and mortality after PCI and also preventing $40 \%$ of new HF cases or worsening of HF in the following 6 months) the price of the cardioprotective agent could be as high as $£ 6660$ and still be below the $£ 20000$ threshold. If the relative risk would only be 0.95 , however, the price should be no higher than $£ 826$. The results of this two-way analysis are shown in figure 4 . The incremental cost-effectiveness ratios at various relative risk and cost values are shown in online supplementary table S1.

In the probabilistic sensitivity analysis, the ICER was below $£ 20000$ per QALY gained in $71 \%$ of the simulations. At a willingness-to-pay of $£ 30000$ per QALY gained, this probability would be $87 \%$. The probability that a cardioprotective agent would be cost-effective at different thresholds is shown in figure 5 .

\section{DISCUSSION}

In this study we have investigated the potential economic consequences of a hypothetical cardioprotective agent.
The results of this study indicate that if a cardioprotective agent could be successfully developed for patients with STEMI to decrease the risk of HF after PCI, it has a high chance of being cost-effective. Currently there are no such cardioprotective agents available, but it is expected that this will change in the near future. In a randomised trial of the cardioprotective effects of hypothermia using cold saline and endovascular cooling the incidence of $\mathrm{HF}$ was significantly lower in the hypothermia group than in the control group (3\% vs $14 \%) .{ }^{25}$ In this trial, no overall effect was shown on infarct size, however, the infarct size was significantly smaller in the subset of patients with anterior STEMI. In a small randomised trial cyclosporine reduced infarct size,${ }^{26}$ but this effect could not be replicated in a more recent larger trial. $^{27}$ The antiplatelet drug abciximab reduced 30-day infarct size when administered intravenously, but no significant effect on HF was demonstrated ${ }^{28}$ Intracoronary administration of this drug did not result in a lower risk of mortality or recurrent infarction, but did result in a lower risk of new $\mathrm{HF}(2.4 \%$ vs $4.1 \%)$ compared to intravenous administration. ${ }^{29}$ The antidiabetic drug exenatide could also reduce infarct size, but only in those reperfused early. ${ }^{30}{ }^{31}$ In a recent trial, patients treated with metoprolol before PCI had a smaller infarct size and higher left ventricular ejection fraction. ${ }^{32}$ These trials all suggest there are many potential cardioprotective agents that could affect the outcome of myocardial infarction. Several reviews on this topic therefore
Figure 3 Tornado diagram showing the effect of uncertainty around the most influential parameters on the incremental cost-effectiveness ratio. HF, heart failure; $\mathrm{PCl}$, percutaneous coronary intervention; QALY, quality-adjusted life-year.
Age (55-75 years)

Cost cardioprotective $(£ 1,000-£ 4,000)$

RR HF after PCI (60-100\%)

RR mortality after $\mathrm{PCI}(60-100 \%)$

Discount rate (1.5-5.5\%)

RR new HF first 6 months (60-100\%)

New HF first month (0\%-10\%)

Mortality after $\mathrm{PCl}(1.8 \%-5 \%)$

Excess mortality class II (0-0.5\%)

HF after PCI (7.4\%-12.6\%)

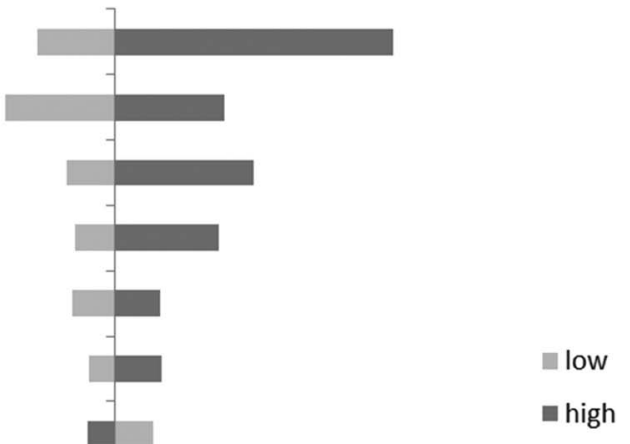

high 


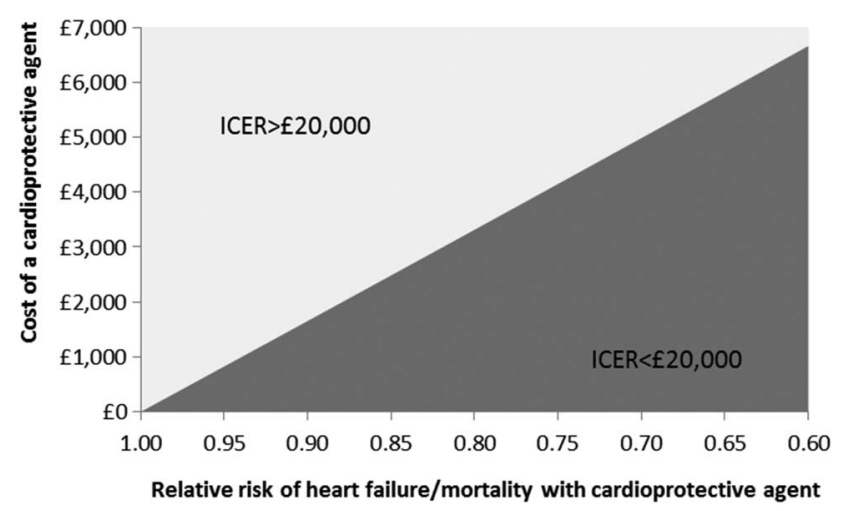

Figure 4 Results of the two-way sensitivity analysis. The ICER would be higher than $£ 20000$ per QALY gained in scenarios represented by the light grey area, while the ICER would be below this threshold in scenarios represented by the dark grey area. ICER, incremental cost-effectiveness ratio; QALY, quality-adjusted life-year.

conclude that it is likely that treatment of reperfusion injury will become available in the near future..$^{2-4}$

The economic burden of HF resulting from AMI has been estimated to be at least $£ 125-181$ million to the NHS and a further $£ 27$ million for nursing home costs in $2000 .^{33}$ The cost-effectiveness of treatment for postmyocardial infarction HF was studied in several countries, such as the USA, Germany, the Netherlands, France and Spain ${ }^{34}$ and also in the UK, where aldosterone antagonists were found to be a highly cost-effective strategy for the management of $\mathrm{HF}^{35}$ However, no studies have been published up to this date on cardioprotective agents to prevent $\mathrm{HF}$ in patients with AMI.

There was considerable uncertainty around many input parameters in the model. This was caused by the lack of specific data about the effectiveness and costs of a cardioprotective agent (due to the hypothetical character of the study) or the small sample size of some studies we used to predict variables in our analysis. We accounted for this uncertainty by varying the parameters over their 95\% CIs or other (wide) plausible range in the probabilistic sensitivity analysis. In this sensitivity

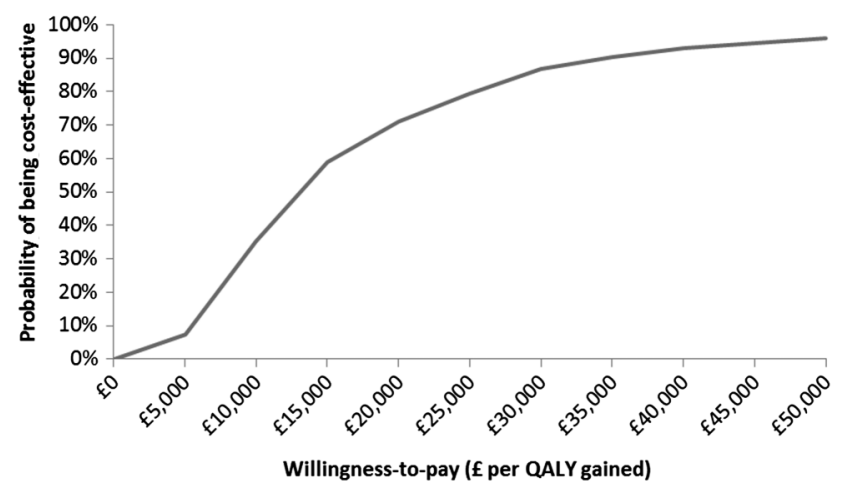

Figure 5 Cost-effectiveness acceptability curve. QALY, quality-adjusted life-year. analysis we performed 1000 simulations to be able to calculate CIs around our results.

The main limitation of this study is that it is based on a hypothetical drug. There is currently not enough data available on the long-term effectiveness of any of the potential cardioprotective agents. We also assumed that the cardioprotective agent would only reduce the risk and severity of $\mathrm{HF}$ and not the risk of other clinical major adverse cardiac events, such as recurrent infarction. In reality, a cardioprotective agent might have an effect on all such events. However, by only taking into account HF risk we present a conservative estimate of the cost-effectiveness. A cardioprotective agent that will also decrease the risk of other major adverse cardiac events is expected to have a higher chance to be costeffective. Future studies should provide more data on the long-term effectiveness of new agents, looking at on-target as well as off-target effects and ideally also at the quality of life. A strength of this study is that a model is presented that can be used for any of the potential cardioprotective agents to study the economic consequences of implementing this agent in clinical practice. If the effect of the new drug on the incidence of HF after PCI is known, this model can be updated to present a more precise estimate of the cost-effectiveness of this drug. For this future research an update of the estimates of relative risks for mortality and HF after PCI is required and if applicable, other benefits of the treatment can be added to the model.

The chance that a new cardioprotective agent will be cost-effective mainly depends on the price of the agent, the age of the patient and the relative risk of HF after PCI. To be cost-effective a higher price for the drug requires a greater effect on HF incidence or mortality. At a price of $£ 2500$ and with a $20 \%$ reduction in $\mathrm{HF}$ incidence and mortality after PCI, it is likely that the cardioprotective agent would be cost-effective.

Acknowledgements The authorsthank Kumar Perampaladas for his useful feedback on the manuscript.

Contributors TIV was involved in the conception and design of the study, data collection, analysis and interpretation of data and drafting of the manuscript. SM was involved in conception and design of the study, interpretation of data, critical revision of the manuscript and supervision. AM was involved in interpretation of data and critical revision of the manuscript. MS was involved in conception and design of the study, interpretation of data and critical revision of the manuscript. All authors approved the final version of the manuscript.

Funding This research received no specific grant from any funding agency in the public, commercial or not-for-profit sectors.

Competing interests None declared.

Provenance and peer review Not commissioned; externally peer reviewed.

Data sharing statement No additional data are available.

Open Access This is an Open Access article distributed in accordance with the Creative Commons Attribution Non Commercial (CC BY-NC 4.0) license, which permits others to distribute, remix, adapt, build upon this work noncommercially, and license their derivative works on different terms, provided the original work is properly cited and the use is non-commercial. See: http:// creativecommons.org/licenses/by-nc/4.0/ 


\section{REFERENCES}

1. Kubler W, Haass M. Cardioprotection: definition, classification, and fundamental principles. Heart 1996;75:330-3.

2. Kloner RA. Current state of clinical translation of cardioprotective agents for acute myocardial infarction. Circ Res 2013;113:451-63.

3. Garcia-Dorado D, Rodríguez-Sinovas A, Ruiz-Meana M, et al. Protection against myocardial ischemia-reperfusion injury in clinical practice. Rev Esp Cardiol (Engl Ed) 2014;67:394-404.

4. Schmidt MR, Pryds K, Bøtker HE. Novel adjunctive treatments of myocardial infarction. World J Cardiol 2014;6:434-43.

5. Cohn JN, Ferrari R, Sharpe N. Cardiac remodeling-concepts and clinical implications: a consensus paper from an international forum on cardiac remodeling. Behalf of an International Forum on Cardiac Remodeling. J Am Coll Cardiol 2000;35:569-82.

6. Sabbah HN, Goldstein S. Ventricular remodelling: consequences and therapy. Eur Heart $J$ 1993;14(Suppl C):24-9.

7. Tisminetzky M, Coukos JA, McManus DD, et al. Decade-long trends in the magnitude, treatment, and outcomes of patients aged 30 to 54 years hospitalized with ST-segment elevation and non-ST-segment elevation myocardial infarction. Am J Cardiol 2014;113:1606-10.

8. Abelin AP, David RB, Gottschall CA, et al. Accuracy of dedicated risk scores in patients undergoing primary percutaneous coronary intervention in daily clinical practice. Can J Cardiol 2014;30:125-31.

9. Kelly DJ, Gershlick T, Witzenbichler B, et al. Incidence and predictors of heart failure following percutaneous coronary intervention in ST-segment elevation myocardial infarction: the HORIZONS-AMI trial. Am Heart J 2011;162:663-70.

10. McManus DD, Chinali M, Saczynski JS, et al. 30-year trends in heart failure in patients hospitalized with acute myocardial infarction Am J Cardiol 2011;107:353-9.

11. Spencer FA, Meyer TE, Gore JM, et al. Heterogeneity in the management and outcomes of patients with acute myocardial infarction complicated by heart failure: the National Registry of Myocardial Infarction. Circulation 2002;105:2605-10.

12. Flather MD, Shibata MC, Coats AJ, et al. Randomized trial to determine the effect of nebivolol on mortality and cardiovascular hospital admission in elderly patients with heart failure (SENIORS). Eur Heart J 2005;26:215-25.

13. Ford E, Adams J, Graves N. Development of an economic model to assess the cost-effectiveness of hawthorn extract as an adjunct treatment for heart failure in Australia. BMJ Open 2012;2:pii: e001094.

14. Yao G, Freemantle N, Flather M, et al. Long-term cost-effectiveness analysis of nebivolol compared with standard care in elderly patients with heart failure: an individual patient-based simulation model. Pharmacoeconomics 2008;26:879-89.

15. Nauta ST, Deckers JW, Akkerhuis KM, et al. Age-dependent care and long-term (20 year) mortality of 14,434 myocardial infarction patients: changes from 1985 to 2008. Int J Cardiol 2013;167:693-7.

16. Scuffham PA, Chaplin S. An economic evaluation of fluvastatin used for the prevention of cardiac events following successful first percutaneous coronary intervention in the UK. Pharmacoeconomics 2004;22:525-35.

17. Department of Health. National schedule of reference costs 2012/13. https://www.gov.uk/government/publications/ nhs-reference-costs-2012-to-2013

18. British National Formulary. https://www.medicinescomplete.com/mc/ bnf/current/

19. Fox M, Mealing S, Anderson $\mathrm{R}$, et al. The clinical effectiveness and cost-effectiveness of cardiac resynchronisation (biventricular pacing) for heart failure: systematic review and economic model. Health Technol Assess 2007;11:iii-iv, ix-248.
20. Briggs $A H$, Ades $A E$, Price MJ. Probabilistic sensitivity analysis for decision trees with multiple branches: use of the Dirichlet distribution in a Bayesian framework. Med Decis Making 2003;23:341-50.

21. Kloner RA, Forman MB, Gibbons RJ, et al. Impact of time to therapy and reperfusion modality on the efficacy of adenosine in acute myocardial infarction: the AMISTAD-2 trial. Eur Heart $J$ 2006;27:2400-5.

22. Lønborg J, Kelbaek H, Vejlstrup N, et al. Cardioprotective effects of ischemic postconditioning in patients treated with primary percutaneous coronary intervention, evaluated by magnetic resonance. Circ Cardiovasc Interv 2010;3:34-41.

23. Serruys PW, Unger F, Sousa JE, et al. Comparison of coronary-artery bypass surgery and stenting for the treatment of multivessel disease. N Engl J Med 2001;344:1117-24.

24. NICE National Institute of Health and Care Excellence. Guide to the methods of technology appraisal 2013. http://www.nice.org.uk/ media/D45/1E/GuideToMethodsTechnologyAppraisal2013.pdf

25. Erlinge D, Götberg M, Lang I, et al. Rapid endovascular catheter core cooling combined with cold saline as an adjunct to percutaneous coronary intervention for the treatment of acute myocardial infarction. The CHILL-MI trial: a randomized controlled study of the use of central venous catheter core cooling combined with cold saline as an adjunct to percutaneous coronary intervention for the treatment of acute myocardial infarction. J Am Coll Cardiol 2014;63:1857-65

26. Piot C, Croisille P, Staat $\mathrm{P}$, et al. Effect of cyclosporine on reperfusion injury in acute myocardial infarction. $N$ Engl J Med 2008;359:473-81.

27. Ghaffari S, Kazemi B, Toluey M, et al. The effect of prethrombolytic cyclosporine-A injection on clinical outcome of acute anterior ST-elevation myocardial infarction. Cardiovasc Ther 2013;31:e34-9.

28. Stone GW, Maehara A, Witzenbichler B, et al. Intracoronary abciximab and aspiration thrombectomy in patients with large anterior myocardial infarction: the INFUSE-AMI randomized trial. JAMA 2012;307:1817-26.

29. Thiele $\mathrm{H}$, Wöhrle J, Hambrecht $\mathrm{R}$, et al. Intracoronary versus intravenous bolus abciximab during primary percutaneous coronary intervention in patients with acute ST-elevation myocardial infarction: a randomised trial. Lancet 2012;379:923-31.

30. Lønborg J, Kelbaek H, Vejlstrup N, et al. Exenatide reduces final infarct size in patients with ST-segment-elevation myocardial infarction and short-duration of ischemia. Circ Cardiovasc Interv 2012;5:288-95.

31. Woo JS, Kim W, Ha SJ, et al. Cardioprotective effects of exenatide in patients with ST-segment-elevation myocardial infarction undergoing primary percutaneous coronary intervention: results of exenatide myocardial protection in revascularization study. Arterioscler Thromb Vasc Biol 2013;33:2252-60.

32. Ibanez B, Macaya C, Sánchez-Brunete V, et al. Effect of early metoprolol on infarct size in ST-segment-elevation myocardial infarction patients undergoing primary percutaneous coronary intervention: the Effect of Metoprolol in Cardioprotection During an Acute Myocardial Infarction (METOCARD-CNIC) trial. Circulation 2013;128:1495-503.

33. Lacey L, Tabberer M. Economic burden of post-acute myocardial infarction heart failure in the United Kingdom. Eur $J$ Heart Fail 2005;7:677-83

34. Croom KF, Plosker GL. Eplerenone: a pharmacoeconomic review of its use in patients with post-myocardial infarction heart failure. Pharmacoeconomics 2005;23:1057-72.

35. McKenna C, Walker S, Lorgelly $P$, et al. Cost-effectiveness of aldosterone antagonists for the treatment of post-myocardial infarction heart failure. Value Health 2012;15:420-8. 